\title{
Contribution of 3-D electrical resistivity tomography for landmines detection
}

\author{
M. Metwaly ${ }^{1,2,5}$, G. El-Qady ${ }^{1}$, J. Matsushima ${ }^{2}$, S. Szalai ${ }^{3}$, N. S. N. Al-Arifi ${ }^{4}$, and A. Taha ${ }^{1}$ \\ ${ }^{1}$ National Research Institute of Astronomy and Geophysics (NRIAG), 11722 Helwan, Cairo, Egypt \\ ${ }^{2}$ Geosystem Engineering Department, Graduate School of Engineering, The University of Tokyo, Japan \\ ${ }^{3}$ Geodetic and Geophysical Research Institute of the Hungarian Academy of Science, University of West Hungary, 9400 \\ Sopron, Csatkai u. 6-8, Hungary \\ ${ }^{4}$ Geology Department, Collage of Science, King Saud University, Saudi Arabia \\ ${ }^{5}$ Al-Quwiy’yia Community College, King Saud University, Saudi Arabia
}

Received: 6 March 2008 - Revised: 21 July 2008 - Accepted: 28 October 2008 - Published: 11 December 2008

\begin{abstract}
Landmines are a type of inexpensive weapons widely used in the pre-conflicted areas in many countries worldwide. The two main types are the metallic and nonmetallic (mostly plastic) landmines. They are most commonly investigated by magnetic, ground penetrating radar (GPR), and metal detector (MD) techniques. These geophysical techniques however have significant limitations in resolving the non-metallic landmines and wherever the host materials are conductive. In this work, the 3-D electric resistivity tomography (ERT) technique is evaluated as an alternative and/or confirmation detection system for both landmine types, which are buried in different soil conditions and at different depths. This can be achieved using the capacitive resistivity imaging system, which does not need direct contact with the ground surface. Synthetic models for each case have been introduced using metallic and non-metallic bodies buried in wet and dry environments. The inversion results using the $\mathrm{L}_{1}$ norm least-squares optimization method tend to produce robust blocky models of the landmine body. The dipole axial and the dipole equatorial arrays tend to have the most favorable geometry by applying dynamic capacitive electrode and they show significant signal strength for data sets with up to $5 \%$ noise. Increasing the burial depth relative to the electrode spacing as well as the noise percentage in the resistivity data is crucial in resolving the landmines at different environments. The landmine with dimension and burial depth of one electrode separation unit is over estimated while the spatial resolutions decrease as the burial depth and noise percentage increase.
\end{abstract}

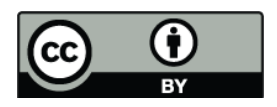

Correspondence to: M. Metwaly (mmetwaly70@yahoo.com)

\section{Introduction}

Landmine contamination is one of most widespread calamities, which transcends humanitarian and sociological concerns and brings severe environmental, economic and development problems. It is not known exactly how many landmines were planted and where these mines were located. Nonetheless, it is estimated that about 80-120 million landmines have already been planted in many post-conflicted areas, in about 90 countries (Berhe, 2007). The areas contaminated with mines directly and indirectly impact the surrounding community. Complete clearance of any landmine field is required to restore public confidence. The fear of the presence of even a single landmine deny people access to large area that is desperately required for agriculture, water supply, and to undertake economical evaluation for the natural resources. Therefore, mine-detection techniques require extremely high detection rates and accuracy. Although research and development of detection techniques has been going on for many years, no single technique is deemed suitable for all types of landmines.

Recently there are numerous efforts to evaluate different technologies for the detection of landmines (Savelyev et al., 2007). Several geophysical techniques have been proposed and utilized worldwide to achieve these objectives. Among these, ground penetrating radar (GPR) and metal detector (MD) are considered the most effective ones, because they can locate both metallic and nonmetallic landmines by noninvasive subsurface sensing (Gao et la., 2000; Chen et al., 2001; Daniels, 2004). However, it is well known that the performance of GPR is influenced by the EM properties of the soil, particularly with increase of the moisture and clay contents (Das et al., 2001; Lopera and Milisavljevic, 2007;

Published by Copernicus Publications on behalf of the European Geosciences Union and the American Geophysical Union. 
Metwaly et al., 2007). In highly conductive soil the electromagnetic waves diffuse quickly therefore the GPR, which utilize high frequency waves is not able to see deeply into the ground. Similarly, the application of MD for the landmine detection sometimes fails when the mines are composed of non-metallic materials and/or the soil contains high concentrations of ferruginous minerals (Lopera and Milisavljevic, 2007).

Therefore there is a strong need for applying another nondestructive surface technique, which is neither completely affected by the landmine materials nor by the EM properties of the soil. Such a proposed technique could be used either in combination with the GPR and MD techniques in routine landmine detection or as an independent confirmation tool for the assurance of landmine cleared areas. These requirements could be satisfied by using the electrical resistivity tomography (ERT) technique, particularly the capacitive resistivity (CR) dynamic system (Benderitter et al., 1994). The CR system is similar to the well-known conventional DC resistivity system with the main difference that the galvanic electrodes are replaced by capacitive sensors (Kuras et al., 2006). The ERT method generally provides low cost and rapid tool for generating spatial models of subsurface physical properties (Chambers, et al., 2006).

The main aim of this work is therefore to investigate the applicability and effectiveness of the 3-D electrical resistivity tomography technique to locate small sized metallic and non-metallic landmines buried in resistive or conductive environments at different depths using different electrode configurations.

\section{Electrical Resistivity Tomography}

The electrical resistivity tomography technique is well known in geoexploration. The electrical potentials are measured at grid points on the ground surface for number of current injection points. Then the apparent electrical conductivity/resistivity is calculated and used to construct the subsurface conductivity image that can be used for identifying any anomalies like landmines. The presence of metallic and non-metallic mines will disturb the subsurface conductivity distributions. The signal characteristics are based on the size, shape, conductivity contrast, and the depth of the buried object. For landmine detection, the main advantage of the ERT method is that it works effectively in wet and conductive environments while many other electromagnetic techniques (GPR and MD) perform poorly. Moreover, the ERT is a low cost technique, which is able to confirm the results of classical clearance operations.

There are few published works for using the ERT for the landmine detection. Recently Church et al. (2006) showed the reliability of the ERT technique for detecting the indoor and outdoor surrogates and passive landmines of various types buried in different soils as well as under shallow water. They used fixed classical electrodes with advanced algorithm for reconstructing the subsurface conductivity distribution at shallow depth. Most of the other trials focused to detect the unexploded ordinance (UXO) using generally 2-D ERT (El-Qady and Ushijima, 2006). However, the UXO are actually not of primarily interest for ERT since it mainly composes of metallic components and could be detected better using the conventional electromagnetic techniques. Metwaly (2007) compared the 2-D GPR and ERT responses of metallic and plastic landmines at different soils however the landmines are in fact 3-D bodies. Therefore, this work is considered one step forward to deal with various small landmines buried at different depths in dry and wet environments using two electrode arrays.

The classical mechanical installation of the steel electrodes is impractical and probably risky when used for landmine detection. Therefore, a need has arisen for alternative resistivity imaging methodology like the capacitive electric resistivity system, which does not need direct coupling with the ground surface. The technique is based on a four-point sensors array that is capacitively coupled to the ground and acts as an oscillating non-grounded electric dipole (Kuras et al., 2007). The coupling mechanism between sensors and the ground is then predominantly capacitive and the inductive effects are negligible. The entire system is designed to be dragged or towed along the ground surface either manually or mechanically while resistivity can be measured continuously (Milsom, 2003). There are basically, two types of capacitive sensors, one is the capacitive line antennae, and the other is flat plate electrodes (Kuras et al., 2007). Under some conditions, the capacitive measurement of resistivity emulates the DC field surveys and different measurements can be employed with the CR system (such as resistivity sounding, profiling, tomographic imaging). Moreover, the apparent resistivity determined using the CR system is processed using the traditional DC interpretation schemes.

\section{Electrodes configuration}

The dipole axial and the dipole equatorial arrays tend to have the most favorable geometry by applying dynamic capacitive electrode (Parasnis, 1997). This is referring to their ease to use and the superior horizontal and vertical resolution relative to the array dimensions (Kuras et al., 2006). The other electrode configurations are generally unsuitable for the towed resistivity capacitive system. This is referring to the using of remote electrodes that need quite long wire connecting cables, which have large capacitance interference between transmitter and receiver. In the current example, we used different numbers of electrodes in both directions constructing a rectangular research model area. The maximum number of independent measurements that can be simply made with $n_{e}$ electrodes is (Xu and Noel, 1993):

$n_{\max }=n_{e}\left(n_{e}-1\right) / 2$ 
Table 1. The physical and geometrical parameters used in the synthetic data modeling (ES unit is equal to electrode separation).

\begin{tabular}{llccc}
\hline & Quality & Dimension (in ES unit) & Depth (in ES unit) & Resistivity (Ohm.m) \\
\hline \multirow{2}{*}{ Landmine } & metallic & 1 & 1 & 0.01 \\
& non-metallic & 1 & 1 & 100 \\
\hline \multirow{2}{*}{ Host soil } & dry & - & & 1000 \\
& wet & - & 5 \\
\hline
\end{tabular}

Although in some case like in landmine detection, acquiring the complete 3 -D data set is not always possible, therefore it is recommended to acquire the data in 2-D parallel profiles along $\mathrm{x}$ direction and then repeat the measurements similarly in y direction eventually using the same electrode positions and spacing. This way of survey helps in reducing the directional bias, which is commonly dominant in typical 2-D measurements (Loke and Barker, 1996). The synthetic data utilized in this work use the 2-D parallel profiles strategy.

\section{The forward modeling}

The response of the 3-D electrical resistivity models used in this work was calculated using the finite-difference method, in which the subsurface is divided into a 3-D mesh (Dey and Morrison, 1979). Accordingly, the resistivity values have been estimated at each cell of the mesh. Electrical resistivity tomography experiments yield a series of voltage measurements in response to number of known input currents. These voltages and currents are related to the subsurface conductivity structures via the following relation:

$\nabla \cdot(-\sigma \nabla \varphi)=I\left(\delta\left(r-r_{s^{+}}\right)-\delta\left(r-r_{s^{-}}\right)\right)$

This equation relates the potential field $(\varphi)$ to the input current $(I)$ through the conductivity structure of the medium $(\sigma)$. The $r_{s^{+}}$and $r_{s^{-}}$are the locations of the positive and negative current sources respectively and $\delta\left(r-r_{s}\right)$ is the dirac delta function, centered at the current source location. In order to obtain the potential distribution in the 3-D space, we used the finite difference approximation to divide the subsurface into finite number of elements and manipulating the resistivity values of each element. After discretizing the earth into a finite number of elements or a mesh system, the following equation is obtained (Sasaki, 1994):

$\mathbf{A}(\sigma) \boldsymbol{u}=\boldsymbol{q}$

Whereas, $\boldsymbol{u}$ is a vector containing the potentials, $\mathbf{A}(\sigma)$ is the forward operating matrix and $\boldsymbol{q}$ is the vector containing the locations of positive and negative current sources. For calculating the nodal potential for a given conductivity model then:

$\boldsymbol{u}=\mathbf{A}^{-1}(\sigma) \boldsymbol{q}$

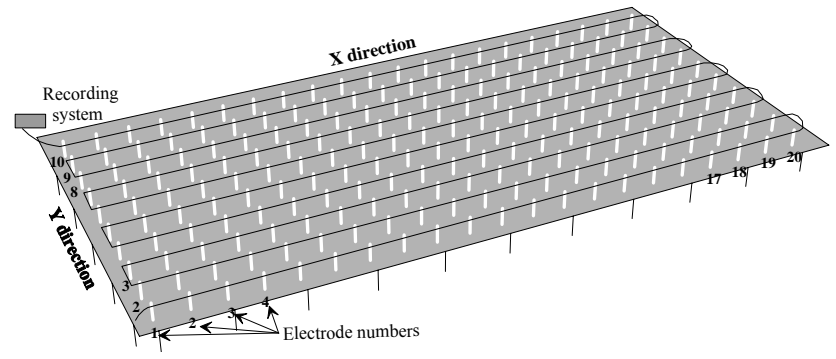

Fig. 1. Electrodes layout used for 3-D synthetic data modeling.

As the nodal potential is now known, the potential differences from point to point and the apparent resistivities are calculated for the applied electrode arrays (Yi et al., 2001).

The landmines and the host soil parameters, which were used to calculate the synthetic data, are summarized in Table 1. The number of employed electrodes is set to be 20 in $\mathrm{x}$ and 10 in y directions (Fig. 1). For both electrode arrays, the dipole length is equal unit electrode spacing (ES), while the dipole separation factor $(n)$ is set to range from $(1 n)$ to $(6 n)$. Both the metallic and non-metallic landmines were modeled with a homogenous cube, whose side length is equal to electrode separation unit (ES) and are buried at depth equal to the electrode separation (ES) as well in homogenous soils. The RES3DMOD $^{1}$ three-dimensional forward modeling program was used to calculating the synthetic apparent resistivity data for both electrode arrays.

\section{The inversion procedure}

\subsection{Inversion scheme}

The fast development of the computer science allows a parallel fast development of automated resistivity inversion routines, which aim to construct the subsurface resistivity distributions in view of data uncertainties (Yi et al., 2001). Among these routines, the regularized least-squares optimization particularly with a smoothness constraint (Sasaki, 1989;

\footnotetext{
${ }^{1}$ RES3DMOD ver. 2.14 3-D resistivity and IP forward modeling using the finite-difference and finite-element methods. www. geoelectrical.com. Accessed: 5.02.2008.
} 
deGroot-Hedlin and Constable, 1990; Loke and Barker, 1996; Li and Oldenburg, 2000; Loke et al., 2003) has become a popular technique for interpreting the ERT data sets. It is considered a flexible method that allows including some constrains during the inversion procedures. Therefore, the resulted inverted models are close to the true subsurface one. There are two broad methods for applying the regularized least-squares optimization technique; the smoothnessconstrained $\mathrm{L}_{2}$ norm and the blocky $\mathrm{L}_{1}$ norm optimization. The $\mathrm{L}_{2}$ norm or smoothness-constrained least squares optimization equation is given by (Loke et al., 2003).

$$
\left(\mathbf{J}_{i}^{T} \mathbf{J}_{i}+\lambda_{i} \mathbf{W}^{r} \mathbf{W}\right) \Delta \boldsymbol{r}_{i}=j_{i}^{T} \boldsymbol{g}_{i}-\lambda_{i} \mathbf{W}^{T} \mathbf{W} \boldsymbol{r}_{i-1}
$$

where $g_{i}$ is the discrepancy data misfit vector containing the difference between the logarithms of the measured and calculated resistivity values, $\Delta \boldsymbol{r}_{i}$ is the change in the model parameters for the $i$ th iteration and $\boldsymbol{r}_{i-1}$ is the model parameters vector for the pervious iteration, $\mathbf{J}$ is the Jacobian matrix of partial derivatives, $\mathbf{W}$ is the roughness filter matrix, $\lambda$ is the damping factor.

In the smooth $\mathrm{L}_{2}$ norm, the sum of squares of the spatial changes in the model resistivity and data misfit is minimized (deGroot-Hedlin and Constable, 1990). On the other hand, the simple method of implementing the $\mathrm{L}_{1}$ norm using the standard least-squares formulation is the iteratively reweighted least-squares method (Wolke and Schwetlick, 1988). Then the optimization equation is modified to be:

$$
\left(\mathbf{J}_{i}^{T} \mathbf{R}_{\mathbf{d}} \mathbf{J}_{i}+\lambda_{i} \mathbf{W}^{r} \mathbf{R}_{\mathbf{d}} \mathbf{W}\right) \Delta \boldsymbol{r}_{i}=j_{i}^{T} \mathbf{R}_{\mathbf{d}} g_{i}-\lambda_{i} \mathbf{W}^{T} \mathbf{R}_{\mathbf{d}} \mathbf{W} \boldsymbol{r}_{i-1}
$$

where $\mathbf{R}_{\mathbf{d}}$ and $\mathbf{R}_{\mathbf{m}}$ are weighting matrixes.

The inversion method using the $\mathrm{L}_{1}$ norm robust method tends to produce models with piecewise constant resistivity values in which the sum of absolute values of the data misfit is minimized (Farquharson and Oldenburg, 1998).

\subsection{Selection of the inversion scheme for the modeling}

Studying the inversion results of synthetic models using different inversion schemes provides good estimations for predicting the features of the near surface small targets like the landmines (Kuras et al., 2006). The 3-D electrical resistivity synthetic data were inverted using RES3DINV commercial software, which offers two inversion schemes using smooth $\mathrm{L}_{2}$ norm and blocky $\mathrm{L}_{1}$ norm implementations of the regularization least-squares optimization method (Loke and Dahlin 2002; Loke et al., 2003; Dahlin and Zhou, 2004). The two inversion schemes were applied separately in order to understand the difference in their behaviors for reconstructing the subsurface and localizing the landmines correctly in various environments. The forward problem was solved using the finite-difference method. The dipole axial electrode array was used as an example for confirming the efficiency of the

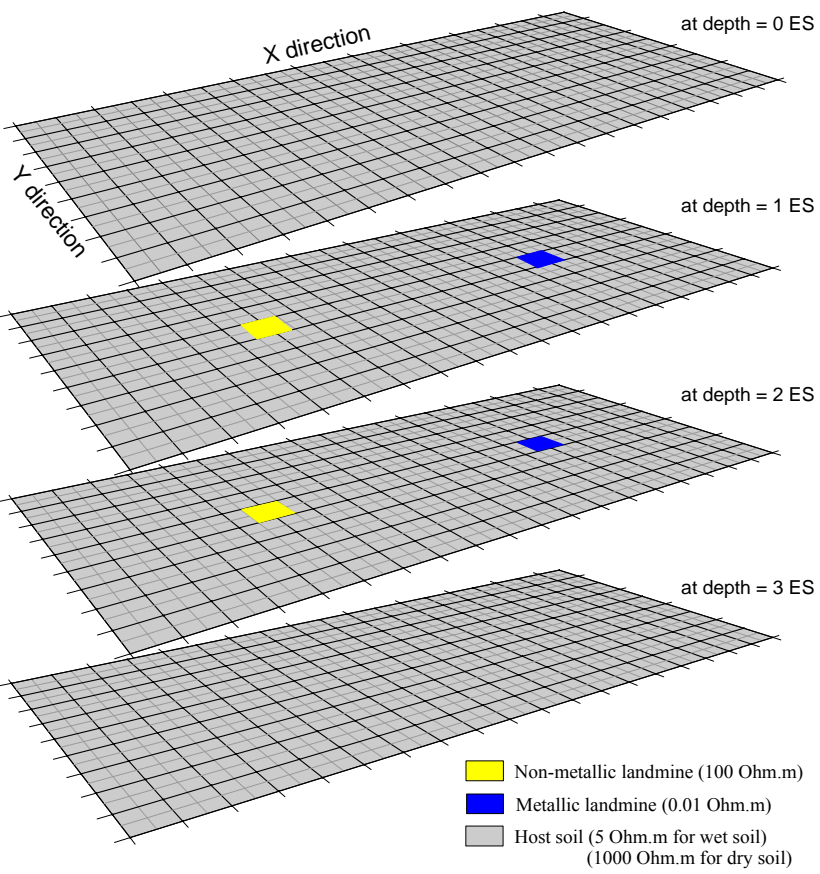

Fig. 2. Model slices for the metallic and non-metallic landmines used in the modeling process. ES is the electrode separation.

inversion schemes, as it has considerable sensitivity to lateral resistivity contrasts (Monteiro Santos et al., 2007; Alaia et al., 2008). The dipole length was equal to one electrode separation unit and the dipole separation was set to range from $n$ to $6 n$. The synthetic models consist of homogenous wet and dry soils with resistivity of $5 \Omega . \mathrm{m}$ and $1000 \Omega$.m respectively having two small metallic and non-metallic landmines buried at depth equal to the unit electrode separation (Table 1). The two landmine types have a resistivity of 0.01 and $100 \Omega$.m respectively (Fig. 2). The 3-D inverted resistivity data using both $\mathrm{L}_{1}$ norm and $\mathrm{L}_{2}$ norm schemes for both wet and dry soil models are displayed as horizontal resistivity images at depth equals to the unit electrode separation (Fig. 3). Both inversion techniques detect the metallic and non-metallic landmines in wet and dry soils successfully, but with different spatial resolutions. The resistivity values of the metallic landmine are relatively lower than the background resistivity in both soil conditions. The non-metallic landmine signature is relatively higher than background resistivity of wet soil and lower than background resistivity of the dry soil (Fig. 3). The inversion results using $\mathrm{L}_{2}$-norm show a type of concentric smeared out anomalies with gradational boundaries around the landmine targets (Fig. 3a and c). The inversion results using $\mathrm{L}_{1}$ norm scheme (Fig. $3 \mathrm{~b}$ and d) give better landmine resolutions in wet and dry soils without such high smearing effect. The inverted images for both metallic and non-metallic bodies' exhibit relatively sharp boundaries between the landmines and the background soils, which are uniformly distributed around the landmines, compared with the $\mathrm{L}_{2}$-norm inversion results. 
(a)

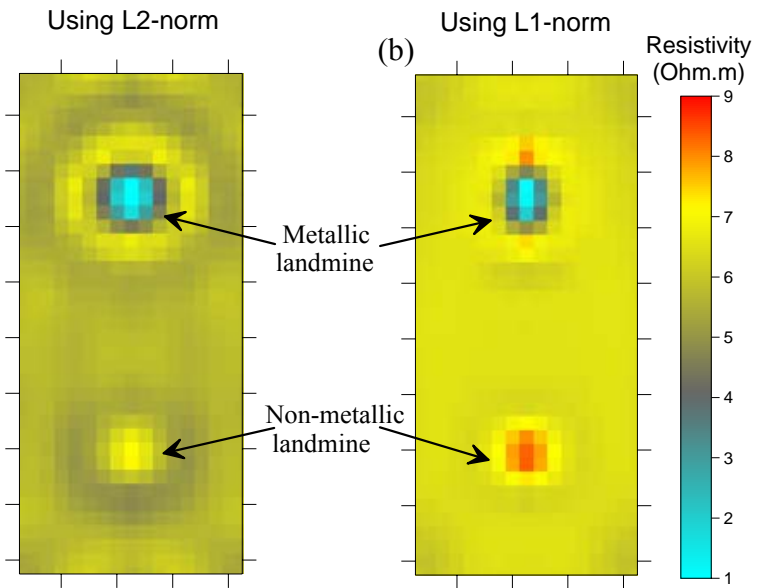

(c)

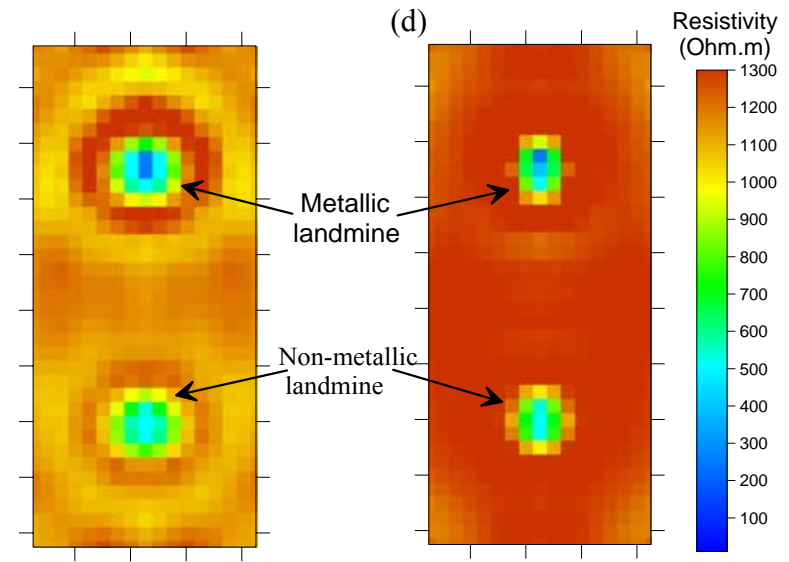

Fig. 3. Horizontal depth slices using the $\mathrm{L}_{1}$ norm and $\mathrm{L}_{2}$ norm $3-\mathrm{D}$ inversion schemes at depth equal to electrode separation. (a) and (b) at the wet soil condition, (c) and (d) at the dry soil condition.

In order to correlate the vertical resistivity resolution of the results using the $\mathrm{L}_{2}$ norm and $\mathrm{L}_{1}$ norm inversion schemes, the vertical resistivity models that pass through the center of the two bodies were extracted and displayed as a function of depth (Fig. 4). The dash-dotted lines show the true landmine and soil models. The continuous line is the inverted model using $\mathrm{L}_{2}$ norm, and the dotted line corresponds to the inverted model using the $\mathrm{L}_{1}$ norm. Neither of the $\mathrm{L}_{2}$ nor $\mathrm{L}_{1}$ norm schemes resolves the original modeled resistivity values of the metallic or non-metallic landmines. This is due to the relative small sizes of the landmines in addition to the relative smoothing effects of both inversion schemes (Loke et al., 2003). However, both inversion schemes give good correlated resistivity values with the soil resistivity particularly underneath the landmine bodies. The vertical resistivity values using the $\mathrm{L}_{1}$ norm inversion scheme are relatively better in representing the true resistivity of both the host soils and the landmines (Fig. 4). The resistivity values show quite high contrast at the boundaries of the landmines compared with the $\mathrm{L}_{2}$ norm inversion results, which are characterized
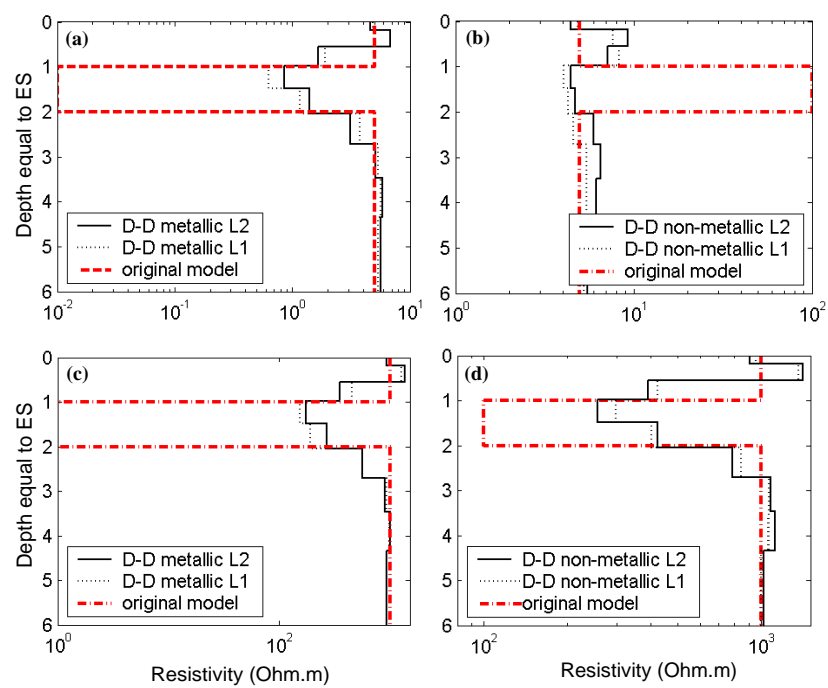

Fig. 4. $L_{1}$ and $L_{2}$ norm resistivity models with depth extracted from the inverted 3-D resistivity data at the center of the landmines. ES is the electrode separations, D-D is the dipole-dipole configuration. (a) and (b) are the metallic and non-metallic landmines in wet soil. (c) and (d) are the metallic and non-metallic landmines in dry soil.

by relative low contrasts and kind of the resistivity oscillations particularly below the landmines. Therefore, based on the horizontal and vertical spatial resolution of the inversion results, the $\mathrm{L}_{1}$ norm inversion scheme will be considered for processing the synthetic 3-D data sets in the coming sections.

\section{Synthetic models}

Certainly, a comprehensive comparison of the near subsurface resistivity imaging abilities using different electrode arrays is required in order to evaluate the suitability of their behavior and resolution for practical landmine imaging applications. Moreover, the optimum fieldwork design, the robust data processing scheme, the spatial resolutions, and the noise sensitivities of the arrays should be known before practical applications. The current model concerns with testing the accuracy of two electrode arrays (dipole axial and dipole equatorial), which are applicable to use with the dynamic capacitive electrode system for the landmine detection. The model consists of metallic and non-metallic landmines with resistivities of 0.01 and $100 \Omega$.m respectively buried in a homogenous conductive (wet) and resistive (dry) soils having resistivity values $5 \Omega$.m and $1000 \Omega$.m respectively. The modeled landmine bodies have a shape of a homogenous cube with a side length equal to the unit electrode separation (ES) and buried at depth equal to the ES as well (Table 1). 

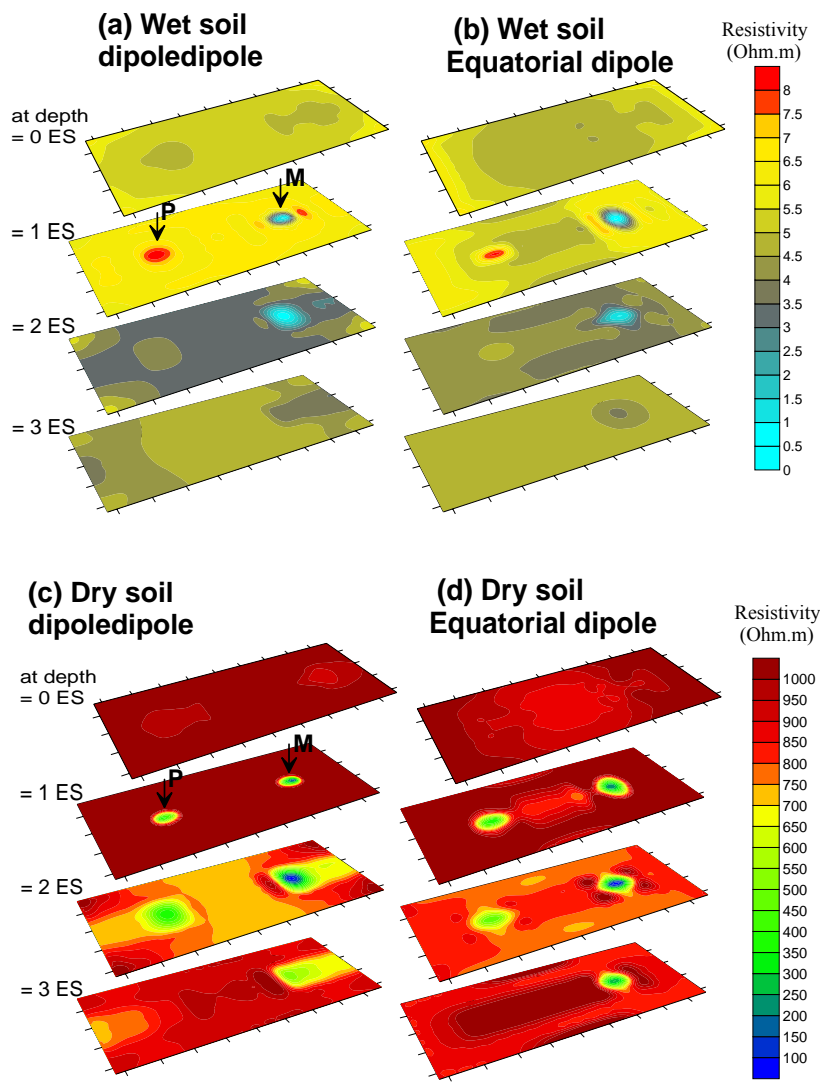

Fig. 5. Inverted models using robust $\mathrm{L}_{1}$ norm for buried metallic and non-metallic landmines in wet and dry environments. ES is the electrode separation. $\mathrm{P}$ and $\mathrm{M}$ are the non-metallic and metallic landmines.

\subsection{Landmines in homogenous soils}

Reasonable reconstructions of the subsurface resistivity distribution were obtained at four different successive depths as a function of unit electrode separation $(0,1,2$, and 3 of the ES). Figure 5 shows the 3-D inversion results using the $\mathrm{L}_{1}$ norm inversion scheme after 6 iterations, which is considered enough for converging the raw synthetic data to the true model. It can be clearly seen that the two electrode arrays detect the location of the metallic and the non-metallic mines either in wet or dry soil with varying spatial resolutions. The conductive metallic landmine has more distinctive resistivity signals compared to the response of resistive non-metallic landmine in both soils. The inverted resistivity anomaly of the metallic landmine is apparently continuous downward below the bottom of the body (Fig. 5). This is due to the current channeling in the metallic landmine rather going down. This effect is significant in resistive soil than in conductive one. On the other hand, the detection of nonmetallic mines in both wet and dry soil is quite major challenge for the other detection techniques. The non-metallic landmine in a wet environment can be detected clearly how-

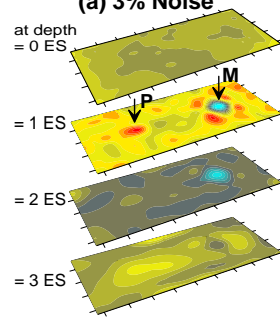

(d) $3 \%$ Noise (a) $3 \%$ Noise

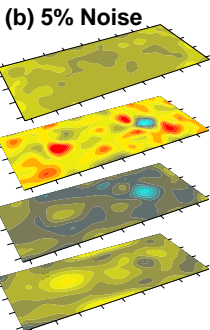

(e) $5 \%$ Noise

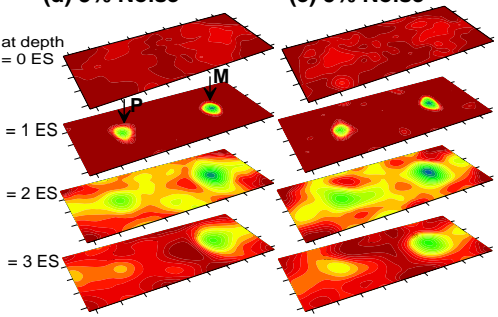

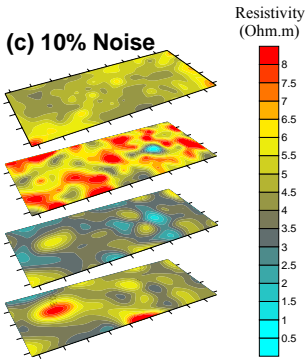

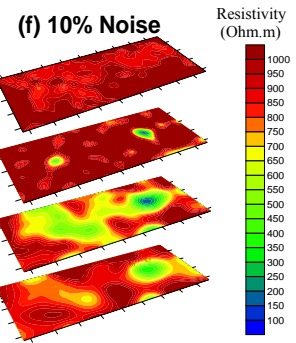

Fig. 6. Inverted models using robust $\mathrm{L}_{1}$ norm for buried metallic and non-metallic landmine in wet and dry environments with different percentage of noise.

ever, the resistivity anomaly from the bottom of the landmine is decrease rapidly. In dry soil, the two electrode arrays sharply portray the top and bottom of the non-metallic landmine. However, the equatorial dipole configuration shows relatively better results (Fig. 5d). In the wet environment, the dipole axial array shows relatively strong resistivity signals (Fig. 5a).

\subsection{The noise effect}

For studying the effect of the noise on the robustness of detecting the different landmines in various environments using ERT technique, Gaussian random noises with amplitudes $3 \%, 5 \%$, and $10 \%$ (Press et al., 1992) were added to the synthetic data. The dipole axial configuration is used for constructing the synthetic model using the same unit electrode separation as in the case of free noise example. These noise levels are of same order or higher than those are observed in the resistivity data acquired for very shallow subsurface investigations (Loke et al., 2003). The inversion results using the 3-D robust $\mathrm{L}_{1}$ norm scheme for both the wet and dry soils are shown in Fig. 6. The resulting models at different depths show slightly distorted resistivity images compared with the noise free data sets in Fig. 5. In wet soil, it is possible to locate the metallic and non-metallic landmines at depths equal to 1 and 2 unit electrode separations as long as the noise level is less than 5\% (Fig. 6a and b). However, the inverted images with high noise level $(10 \%)$ are quite distorted particularly at shallow depths equal to 0 and 1 unit electrode separations (Fig. 6c). Neither the metallic nor the non-metallic landmine can be clearly detected. Conversely in the case of dry 


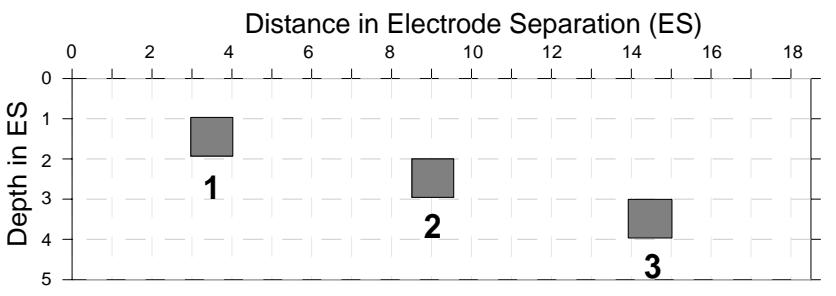

Fig. 7. Synthetic model of three landmines at different depths in homogenous soil.

soil the resistivity contrast is rather higher than the case of wet soil, therefore the resistivity responses from metallic and non-metallic mines are significant even in the case of high noise amplitude (10\%), (Fig. 6d, e, and f). The top of both metallic and non-metallic landmines are clearly resolved at a depth equal to the unit electrode separation. The bottoms of the two landmines are partially blurred at depths equal to 2 and 3 unit electrode separations.

\subsection{Landmines at different depths}

To investigate the capability of the electrical resistivity technique for detecting metallic and non-metallic landmine bodies at various depths, Fig. 7 shows three bodies with symmetrical dimensions equal to the unit electrode separation buried at three different depths equal to 1,2 and 3 unit electrode separations. The resistivity of the landmine bodies and soils are changed to represent the different cases of wet and dry soils as well as the metallic and non-metallic landmines (Table 1). The dipole axial array configuration is applied for creating the synthetic resistivity data of three metallic and non-metallic landmines at different depths.

Figure 8 illustrates the inverted models using the robust $\mathrm{L}_{1}$ inversion scheme for the three metallic and non-metallic landmines buried at three depths in wet and dry soils. The three metallic landmines can be identified correctly in both wet and dry soils with different spatial resolutions. The inverted resistivity images in wet soil are characterized by relative artifacts particularly at the side where the metallic landmines are buried deeper (Fig. 8a). In the case of dry soil, the inverted resistivity images are quite clear. The three metallic landmines produce significant resistivity anomalies, which tend to be slightly smeared with the increasing burial depth (Fig. 8d). Nevertheless, as we mentioned early, the detection of metallic landmines is best performed using the classical metal detector (MD) and magnetic techniques. The detection of non-metallic landmines at various depths and different soils show quite clear resistivity images (Fig. 8). The first two non-metallic landmines at depths equal to 1 and 2 unit electrode buried in both soil types would be detected (Fig. 8b and e). The third non-metallic mines at a depth equal to 3 times unit electrode separation does not cause any significant resistivity anomaly neither in wet nor in dry soil.
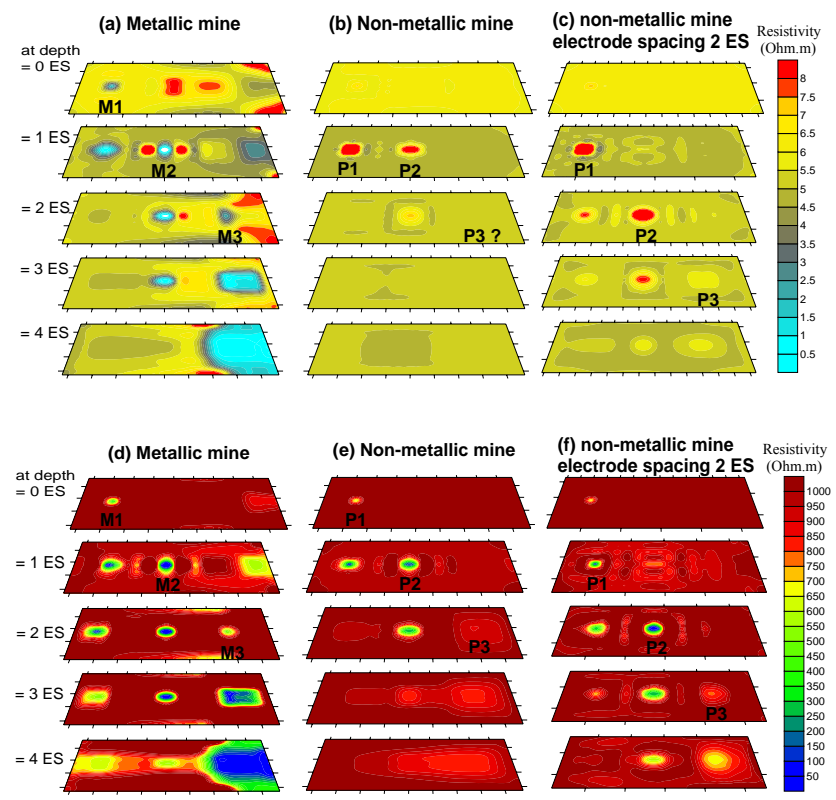

Fig. 8. Inverted models using robust $\mathrm{L}_{1}$ norm inversion for metallic (M) and non-metallic (P) landmines in wet and dry soils. ES is the electrode separation.

Consequently, the applied electrode configuration falls to reconstruct them. This means also that the current dipole axial configuration gives similar penetration depths in both soil types. One possible solution to enhance the spatial resolution of the ERT technique to detect such small non-metallic landmine at that depth is to set the landmine dimension equal to half the unit electrode separation. The inverted models using the double electrode separation show better images for locating the non-metallic landmine at such depth as well as the other two shallow landmines particularly in the dry soil, (Fig. 8c and f).

\subsection{Spatial resolution}

We investigated the effect of noise on the spatial resolution of the ERT method applying for detecting landmines at different depths. Three different Gaussian random noise amplitudes $(3 \%, 5 \%$, and $10 \%)$ have been added to the synthetic data of the previous example. In this case, the landmine dimension is half the unit electrode separation of the dipole axial configuration. The resulted $3-\mathrm{D}$ inversed images using the $\mathrm{L}_{1}$ norm scheme at five depth levels $(0,1,2,3$, and $4 \mathrm{ES})$ are quite distorted (Figs. 9 and 10) in comparison with the noise free data in Fig. 8. In spite of the various noise amplitudes in the resistivity data of the wet and dry soils, the metallic landmines at the three depth levels show significant resistivity anomalies starting from 2 ES unite depth (Figs. 9 and 10). The smearing effects of the inversion scheme are more noticeable at the shallow depth in wet soil than in dry soil and diminish as the investigation depth increases. At 


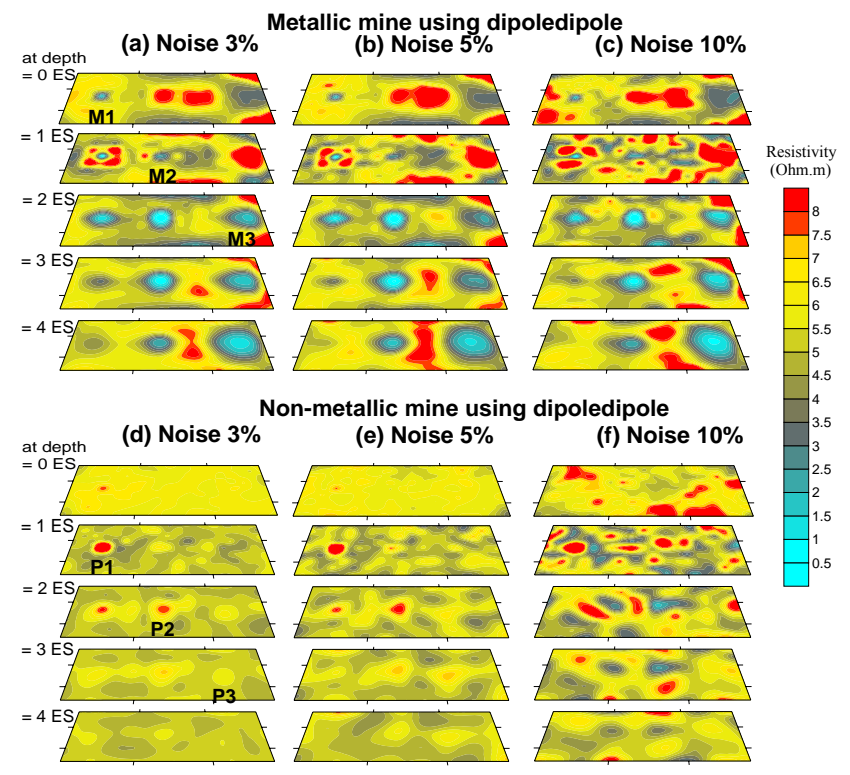

Fig. 9. Inverted models using robust $\mathrm{L}_{1}$ norm inversion for metallic and non-metallic landmines in wet soil.

low noise levels $(\leq 5 \%)$ the inverted resistivity images of the wet and dry soils have quite superior reconstruction of metallic landmines at the three depths (Figs. 9 and 10). With increasing noise amplitude to $10 \%$ the inverted images show quite distorted anomalies, although the metallic landmines nonetheless can be identified in both soils.

In contrary, the inversion of contaminated resistivity data acquired in wet and dry soils, which contain non-metallic landmines give relatively quite clean results (Figs. 9 and 10). In wet and dry environments, the non-metallic landmines at shallow depths (equal to 1 and 2 unit ES) produce clear resistivity anomalies in the inverted images as long as the noise less than $5 \%$. The top and bottom of the landmines can be clearly identified (Figs. 9d and e; 10d and e). The deeper non-metallic landmine at depth equal to 3 unit ES has weak indications in both soils. With increasing the noise level to $10 \%$, neither the shallow nor the deep buried non-metallic landmines can be efficiently detected in both wet and dry environments (Figs. 9f and 10f). However, the shallow nonmetallic landmines in dry soil have better signals relative to the wet soil example.

\section{Conclusions}

In this work, we tested the applicability of electrical resistivity tomography (ERT) technique to detect landmines in different soil conditions and at various depths. Metallic and non-metallic landmines buried in wet and dry soils had been synthetically modeled. Two electrode configurations (dipole axial and dipole equatorial) that are applicable to use with the towed capacitive electrode system were tested to choice

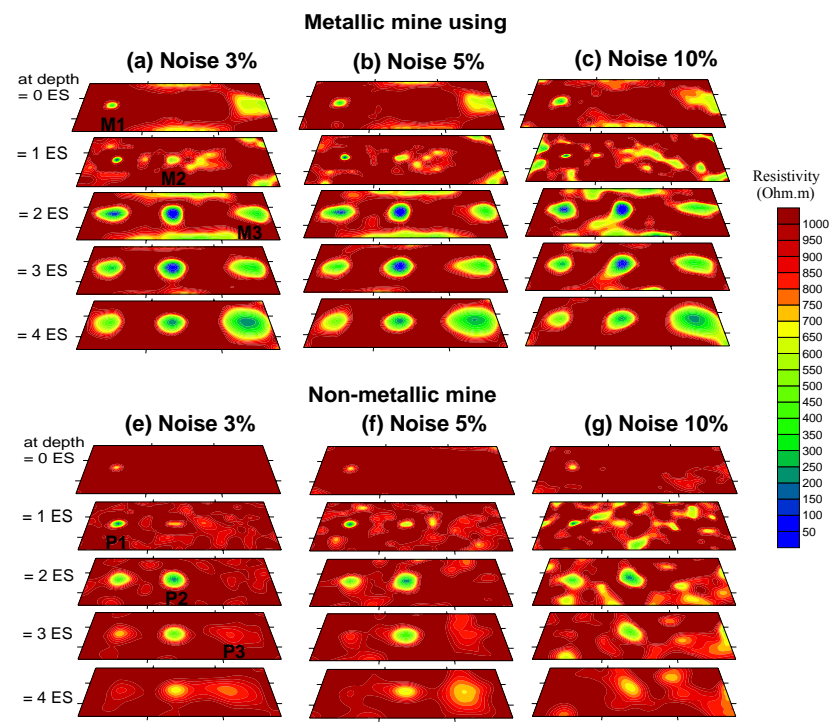

Fig. 10. Inverted models using robust $L_{1}$ norm inversion for metallic and non-metallic landmines in dry soil.

the optimum acquisition parameters. The electrode separations were set to be a function of landmine dimensions, while the buried depths ranged between 1 to 3 times unit electrode separations. From the numerical simulations that were carried out using the 3-D imaging technique, we summarize the success and failures below.

The inversion results using the robust $\mathrm{L}_{1}$ norm leastsquares optimization method tended to produce relatively sharp resistivity images of the landmines. The applied electrode arrays were able to detect the metallic and non-metallic landmines either in wet or dry soils. In wet soil, only the dipole axial array could locate all the metallic and nonmetallic landmines while in dry soil the dipole equatorial configuration gave the clearest images. Therefore, the dipole axial array was selected for other tests. The inversion of contaminated resistivity data with different noise amplitudes acquired for metallic and non-metallic landmines in different soil conditions and at various depths have been tested. According to the inverted resistivity data using the dipole axial array in wet environment, it was possible to locate the metallic and non-metallic landmines as long as the noise level was about $5 \%$. The inverted images with high noise levels (10\%) were distorted and neither the metallic nor the non-metallic landmines could be clearly detected. Conversely, in dry soil even if the resistivity data was highly contaminated with $10 \%$ of noise amplitudes, the inversion results clearly showed the location of metallic and non-metallic landmines.

Another test showed the capability of the ERT technique to locate landmines buried in different environments at three depths. The depths of the metallic and non-metallic landmines were set to be equal to 1 and 2 unit electrode separations. Both landmine types in the wet and dry soils could be 
clearly detected. However, with increasing the buried depth the inversion results showed quite distorted images. One possible solution for increasing the spatial resolution at this depth was to increase the unit electrode separation relative to the landmine dimension.

Similarly, the inversion of noisy data for the same models and parameters showed that the metallic mines at the three depth levels gave significant anomalies in both soil types. However, the non-metallic landmines in wet and dry soils could be detected as long as the depth is not greater than the double the electrode separations and the noise level is lower than $10 \%$. As either the buried depth or the noise level increased, only the shallow non-metallic landmines could be detected in dry soil.

Based on the previous synthetic experiments, we conclude that: 1) the greatest advantage of the electrical technique (ERT) in the field of landmine is that it works well in wet environment where the other detection techniques like MD and GPR are perform poorly. 2) The ERT technique is able to work effectively together with the classical landmine prospecting tools or at least as a confirming tool for the suspicious landmine cleared areas. 3) The horizontal spatial resolution of the resistivity is actually a function of the electrode density and reconstruction depth. Therefore, the landmine targets with dimensions greater than the unit electrode separation and buried at depth equal or less than the unit electrode separation have overestimated spatial resolution.

Acknowledgements. The work of the first author was supported by postdoctoral fellowship of Japanese Society of the Promotion of Science (JSPS). The authors gratefully acknowledge the constructive advices and guidance of anonymous reviewer that substantially improved the manuscript.

Edited by: U. Feudel

Reviewed by: M. Loke and F. Santos

\section{References}

Alaia, R., Patella, D., and Mauriello, P.: Applied of geoelectrical 3D probability tomography in a test-site of the archeological park of Pompei, J. Geophys. Eng., 5, 67-76, 2008.

Benderitter, Y., Jolivet, A., Mounir, A., and Tabbagh, A.: Application of the electrostatic quadripole to sounding in the hectometric depth range, J. Appl. Geophys., 31, 1-6, 1994.

Berhe, A. A.: The contribution of Landmines to land degradation, Land Degrad. Dev., 18, 1-15, 2007.

Chambers, J. E., Kuras, O., Meldrum, P., Ogilvy, R. D., and Hollands, J.: Electrical resistivity tomography applied to geologic, hydrologic, and engineering investigations at a former wastedisposal site, Geophysics, 71, B231-B239, 2006.

Chen, C., Rao, K., and Lee, R.: A tapered-permittivity rod antenna for ground penetrating radar applications, J. Appl. Geophys., 47, 309-316, 2001.

Church, P., McFee, J. E., Gagnon, S., and Wort, P.: Electrical impedance tomographic imaging of buried landmines, IEEE T. Geosci. Remote, 44, 2407-2420, 2006.
Dahlin, T. and Zhou, B.: A numerical comparison of 2-D resistivity imaging with 10 electrode arrays, Geophys. Prospect., 52, 379398, 2004.

Gao, P., Collins, L., Garber, P., Geng, N., and Carin, L.: Calssification of landmine-like metal targets using wideband electromegnatic induction, IEEE T. Geosci. Remote, 23, 35-46, 2000.

Daniels, D.: Surface Penetrating Radar, 2nd edition. The Inst. Electrical Eng., London, 2004.

Das, B., Hendrickx, J., and Borchers, B.: Modeling transient water distributions around landmines in bare soils, J. Soil Sci., 166, 163-173, 2001.

deGroot-Hedlin, C. and Constable, S.: Occam's inversion to generate smooth two-dimensional models from magnetotelluric data, Geophysics, 55, 1613-1624, 1990.

Dey, A. and Morrison, H. F.: Resistivity modeling for arbitrarily shaped three dimensional structures, Geophysics, 44, 753-780, 1979.

El-Qady, G. and Ushijima, K.: Detection of UXO and landmines using 2-D modeling of geoelectrical resistivity data, Proceedings of SAGEEP Meeting, 1176-1182, 2005.

Farquharson, C. G. and Oldenburg, D. W.: Non-linear inversion using general measures of data misfit and model structure, Geophys. J. Int., 134, 213-227, 1988.

Kuras, O., Beamish, D., Melrum, P., and Ogivly, R.: Fundamental of the capacitive resistivity technique, Geophysics, 71, 135-152, 2006.

Kuras, O., Meldrum, P. I., Beamish, D., Ogilvy, R., and Lala, D.: Capacitive resistivity imaging with towed arrays, J. Environ. Eng. Geoph., 12, 267-279, 2007.

Li, Y. and Oldenburg, D. W.: 3-D inversion of induced polarization data, Geophysics, 65, 1931-1945, 2000.

Loke, M. H., Acworth, I., and Dahlin, T.: A comparison of smooth and blocky inversion methods in 2-D electric imaging surveys, Exploration Geophysics, 34, 182-187, 2003.

Loke, M. H. and Barker, R. D.: Practical techniques for 3-D resistivity surveys and data inversion, Geophysics, 44, 499-523, 1996.

Loke, M. H. and Dahlin, T.: A comparison of the Gauss-Newton and quasi-Newton methods in resistivity imaging inversion, J. Appl. Geophys., 49, 149-162, 2002.

Lopera, O. and Milisavljevic, N.: Prediction of the effects of soil and target properties on the antipersonal landmine detection performance of ground-penetrating radar: A Colombian case study, J. Appl. Geophys., 63, 13-23, 2007.

Metwaly, M.: Detection of metallic and plastic landmines using the GPR and 2-D resistivity techniques, Nat. Hazards Earth Syst. Sci., 7, 755-763, 2007, http://www.nat-hazards-earth-syst-sci.net/7/755/2007/.

Milsom, J.: Field Geophysics, 3rd Ed. (The geological field guide series), John Wiley and Sons Ltd, p. 232, 2003.

Monteiro Santos, F. A, Andrade, A. R., and Dupis, A.: 2-D joint inversion of $\mathrm{dc}$ and scalar audio-magnetotelluric data in the evaluation of low enthalpy geothermal fields, J. Geophys. Eng. 4, 53-62, 2007.

Parasnis, D. S.: Principles of applied geophysics, 5th Ed., Chapman and Hall, 1997.

Press, W. H., Teukolsky, S. A, Vetterling, W. T., and Flannery, B. P.: Numerical Recipes in C, 2nd Ed., Cambridge University Press, 1992. 
Sasaki, Y.: Two-dimensional joint inversion of magnetotelluric and dipole-dipole resistivity data, Geophysics, 54, 174-187, 1989.

Sasaki, Y.: 3-D resistivity inversion using the finite element method, Geophysics, 59, 1839-1848, 1994.

Savelyev, T. G., Kempen, L., Sahli, H., Sachs, J., and Sato, M.: Investigation of Time-Frequency Features for GPR landmine discrimination, IEEE T. Geosci. Remote, 45, 118-128, 2007.

Wolke, R. and Schwetlick, H.: Iteratively reweighted least squares algorithms convergence analysis and numerical comparisons, SIAM J. Sci. Stat. Comp., 9, 907-921, 1988.
$\mathrm{Xu}$, B. and Noel, M.: On the completeness of data sets with multielectrode systems for electrical resistivity survey, Geophys. Prospect., 41, 791-801, 1993.

Yi, M., Kim, J., Song, Y., Cho, S., Chung, S., and Suh, J.: Threediminsionaldimensional imaging of subsurface structures using resistivity data, Geophys. Prospect., 49, 483-497, 2001. 